\title{
Glycyrrhizic acid alters Kaposi sarcoma- associated herpesvirus latency, triggering p53-mediated apoptosis in transformed B lymphocytes
}

\author{
Francesca Curreli,1 Alvin E. Friedman-Kien,1,2 and Ornella Flore ${ }^{1,2}$ \\ 1Department of Microbiology and 2Department of Dermatology, New York University School of Medicine, New York, New York, USA.
}

\begin{abstract}
Kaposi sarcoma-associated herpesvirus (KSHV) is linked with all clinical forms of Kaposi sarcoma and several lymphoproliferative disorders. Like other herpesviruses, KSHV becomes latent in the infected cells, expressing only a few genes that are essential for the establishment and maintenance of its latency and for the survival of the infected cells. Inhibiting the expression of these latent genes should lead to eradication of herpesvirus infection. All currently available drugs are ineffective against latent infection. Here we show, for the first time to our knowledge, that latent infection with KSHV in B lymphocytes can be terminated by glycyrrhizic acid (GA), a triterpenoid compound earlier shown to inhibit the lytic replication of other herpesviruses. We demonstrate that GA disrupts latent KSHV infection by downregulating the expression of latency-associated nuclear antigen (LANA) and upregulating the expression of viral cyclin and selectively induces cell death of KSHV-infected cells. We show that reduced levels of LANA lead to p53 reactivation, an increase in ROS, and mitochondrial dysfunction, which result in $\mathrm{G}_{1}$ cell cycle arrest, DNA fragmentation, and oxidative stress-mediated apoptosis. Latent genes are involved in KSHV-induced oncogenesis, and strategies to interfere with their expression might prove useful for eradicating latent KSHV infection and have future therapeutic implications.
\end{abstract}

\section{Introduction}

During herpesvirus latency no virus replication occurs and the viral genome itself persists intact. Such "long term parking" of a viral genome is significant for its stability, which requires a delicate balance among the regulators of viral and cellular gene expression. Herpesviruses modify the expression of cellular genes that regulate apoptosis to meet their requirement for prolonged survival of infected cells, and only a restricted set of viral gene products are made during latent infection. In Kaposi sarcoma-associated herpesvirus (KSHV), a cluster of 3 latent genes (ORF72, ORF73, and $\mathrm{K} 13)$ is transcribed from the same promoter on a polycistronic transcript of $6 \mathrm{~kb}$ designated LT1, which encodes latency-associated nuclear antigen (LANA), viral cyclin (v-cyclin) and viral FLICEinhibitory protein (v-FLIP), and a spliced bicistronic transcript of $2 \mathrm{~kb}$ designated LT2, which encodes $\mathrm{v}$-cyclin and v-FLIP $(1,2)$. ORF73 encodes LANA (3), a multifunctional protein that plays an essential role in the persistence of $\operatorname{KSHV}(4,5)$. LANA interacts with p53 protein, a potent tumor suppressor and cell cycle regulator, by inhibiting its transcriptional activity as well as p53-induced apoptosis $(6,7)$. Loss of p53 function is a frequent and important occurrence in the genesis and progression of many human malignancies. Mitochondria are central death regulators in response to many cytotoxic stimuli and are critical for p53-dependent death,

Nonstandard abbreviations used: AIF, apoptosis-inducing factor; CBX, carbenoxolone; CDK, cyclin-dependent kinase; FLIP, FLICE-inhibitory protein; GA, glycyrrhizic acid; KS, Kaposi sarcoma; KSHV, Kaposi sarcoma-associated herpesvirus; LANA, latency-associated nuclear antigen; TPA, 12-O-tetradecanoyl-phorbol-13acetate; v-, viral.

Conflict of interest: The authors have declared that no conflict of interest exists.

Citation for this article: J. Clin. Invest. 115:642-652 (2005)

doi:10.1172/JCI200523334. because p53 kills cells predominantly via the mitochondrial death pathway. When mitochondria receive a death signal, the outer membrane undergoes permeabilization and releases proteins that promote apoptosis (8); proteases, called caspases, mediate apoptosis (9). Caspases are produced as inactive zymogens but can become activated by a proteolytic cascade by which caspases activate themselves and each other. One of the proteins released by the mitochondria is cytochrome $c$, which, in a complex with the cytoplasmic protein Apaf-1, activates the caspase cascade (10). Mitochondria also release apoptosis-inducing factor (AIF), which, in contrast to cytochrome $c$, triggers apoptosis in a caspase-independent fashion. AIF is a mitochondrial oxidoreductase that in stress conditions translocates from the mitochondria to the nucleus, leading to DNA loss and chromatin condensation; these alterations are observed in cell death when caspases are inhibited $(11,12)$.

Cellular cyclins regulate cell proliferation and cell progression $(13,14)$. All cyclins function by associating with cyclin-dependent kinases (CDKs) to phosphorylate and inactivate cell cycle checkpoint molecules. The KSHV cyclin mainly acts similarly to cellular cyclins. Unlike cellular cyclins, v-cyclin/cdk6 complexes are resistant to the CDK inhibitors p16, p21, and p27 $(15,16)$. $\mathrm{v}$-cyclin/cdk6 complexes phosphorylate p27, inducing its degradation $(16,17)$, and $\mathrm{Bcl}-2$, resulting in its inactivation (18). The abnormal expression of cellular D-type cyclins is strongly implicated in the pathogenesis of various malignancies $(13,19)$. In vitro overexpression of cyclin D induces apoptosis (20); similarly, overexpression of $\mathrm{v}$-cyclin triggers apoptosis in cells with high levels of cdk6 by activating caspase-3 (21).

$\mathrm{v}$-FLIP is encoded by ORF K13 on latency-expressed polycistronic transcripts together with v-cyclin and LANA. Translation of v-FLIP occurs through an internal ribosome entry site present in the 

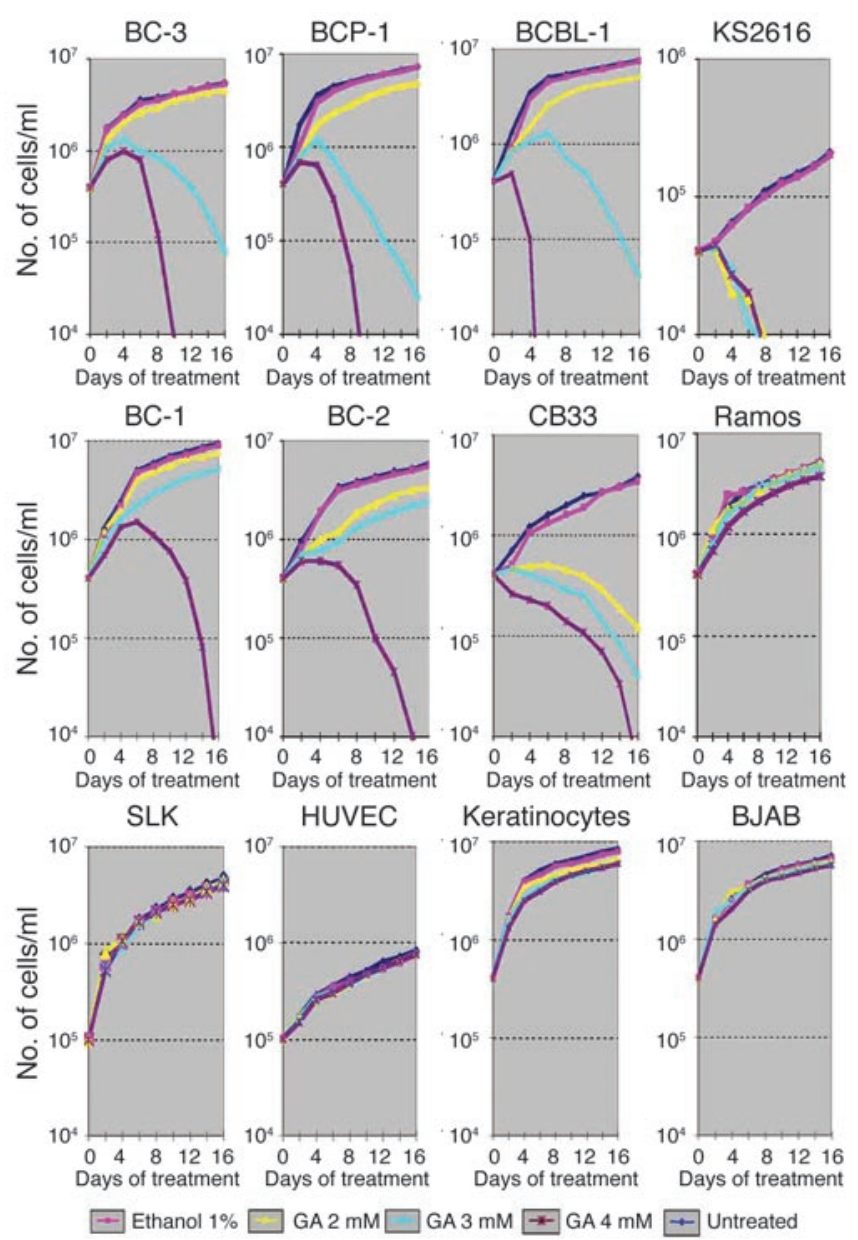

E

$\mathrm{GA} 2 \mathrm{mM} \square$

ORF72 gene (22-24). Cells expressing v-FLIP are protected against apoptosis induced by $\mathrm{CD} 95$ or by tumor necrosis factor receptor- 1 (TNFR-1) $(25,26)$. KSHV-FLIP inhibits Fas-mediated apoptosis through binding procaspase- 8 and preventing its maturation $(27,28)$, indicating that it may play an important role in preventing NK cells from killing KSHV-infected cells. Therefore, the 3 latent genes LANA, v-cyclin, and v-FLIP are crucial for Kaposi sarcoma (KS) and primary effusion lymphoma pathogenesis and might also play an important role in KSHV-induced cell transformation.

Several studies demonstrated that triterpenoid compounds, such as glycyrrhizic acid (GA), have a strong antiviral effect, particularly on herpesviruses. GA treatment of cells infected with herpes simplex virus 1 reduced the infectious viral yield by a factor of more than 3,400-225,000 in a dose-dependent manner (29-31). In addition, GA and its derivatives were shown to be effective both in vitro and in vivo against herpes simplex virus 2 (29-31), varicella zoster $(31,32)$, human $\operatorname{CMV}(31,32)$, and EBV. GA has no effect on EBV adsorption and on particle inactivation but interferes with an early step of viral replication (33). Recently it has been shown that in vitro, GA was the most active compound in inhibiting the replication of the SARS-associated coronavirus (34). Chemically, GA is a conjugate of 1 molecule of the triterpene enoxolone and 2 molecules of glucuronic acid. The hydrophobic triterpenoid structure became the basis for the design of a family of related compounds including GA, carbenoxolone (CBX), and cicloxolone sodium. GA and CBX are estab-

\section{Figure 1}

Proliferation rate of latent KSHV-infected cells in the presence of GA. All cells counts were performed in triplicate and each experiment was repeated 3 times. Cell viability was assessed by counting trypan blueexcluding cells on a hemocytometer every 48 hours. One representative experiment is shown.

lished therapeutic agents in clinical practice; GA is now routinely used throughout Japan for the treatment and control of chronic viral hepatitis, and its transaminase-lowering effect is clinically well recognized (35-37). Several pharmacological actions of GA, such as an antiinflammatory effect $(38,39)$, are considered to be mechanisms by which GA lowers transaminase levels. In human T cell lines, GA treatment enhances Fas-mediated apoptosis without alteration of caspase-3-like activity (40).

The discovery and development of antiherpesvirus drugs able to inhibit productive infection has significantly increased. Unfortunately, the same cannot be said for drugs that target latent herpesvirus infection. To eradicate latent herpesvirus infection, proteins required for maintaining the virus in its latent state must be the target. Because these viral proteins do not include those required for lytic infection, all currently available drugs are ineffective against latent infection. Therefore, there is still a need for a therapeutic agent that can inhibit the persistence of the latent viral status. To date, owing to the absence of latency inhibitors, each KSHV latent gene has been studied separately by expressing it in cellular systems that lack the entire viral genome; for this reason, the results might not reflect what occurs in vivo in KSHV-infected patients. Our experiments were performed in cells derived from KSHV-infected patients, which carry multiple viral copies. This allowed us to study latency disruption by GA in the presence of the entire viral genome.

GA has been shown to be an inhibitor of lytic herpesvirus replication, but its effect against latent herpesvirus infection has never been assessed. The aims of this study were to investigate whether GA could interfere with the latent viral infection by targeting KSHV latent proteins and to determine the biological consequence of latency disruption in cells infected with multiple copies of KSHV. We demonstrate here an important role for GA in disrupting latent KSHV infection by downregulating the expression of LANA and upregulating the expression of v-cyclin. Reduced levels of LANA lead to p53 reactivation, an increase in ROS, and mitochondrial dysfunction, which result in $\mathrm{G}_{1}$ cell cycle arrest and apoptosis of latent KSHV-infected cells.

\section{Results}

Proliferation rate of latent $K S H V$-infected cells in the presence of GA. First we evaluated the cytotoxicity of GA (32). Twelve human cell types, including primary and transformed cells, either uninfected or infected with KSHV, were treated with $0.5,1,2,3$, 4,5 , or $6 \mathrm{mM} \mathrm{GA}$, and its toxicity was assessed by analyzing viability and proliferation rate of treated versus untreated cells by counting trypan blue-excluding cells. Figure 1 shows the growth curves obtained with 2,3, and 4 mM GA; profiles obtained with 0.5 and $1 \mathrm{mM}$ were similar to those obtained with $2 \mathrm{mM} \mathrm{GA}$; 5 and $6 \mathrm{mM}$ GA was toxic for all cell lines, either uninfected or KSHV infected, after 2-4 days of treatment. Within 4 days, the growth curves of all cell cultures, untreated or treated with only $1 \%$ ethanol, increased from $4 \times 10^{5}$ to $2-4 \times 10^{6}$ cells $/ \mathrm{ml}$ for all B cells, from $5 \times 10^{4}$ to $8 \times 10^{4}$ cells $/ \mathrm{ml}$ for KS2616 cells, and from 


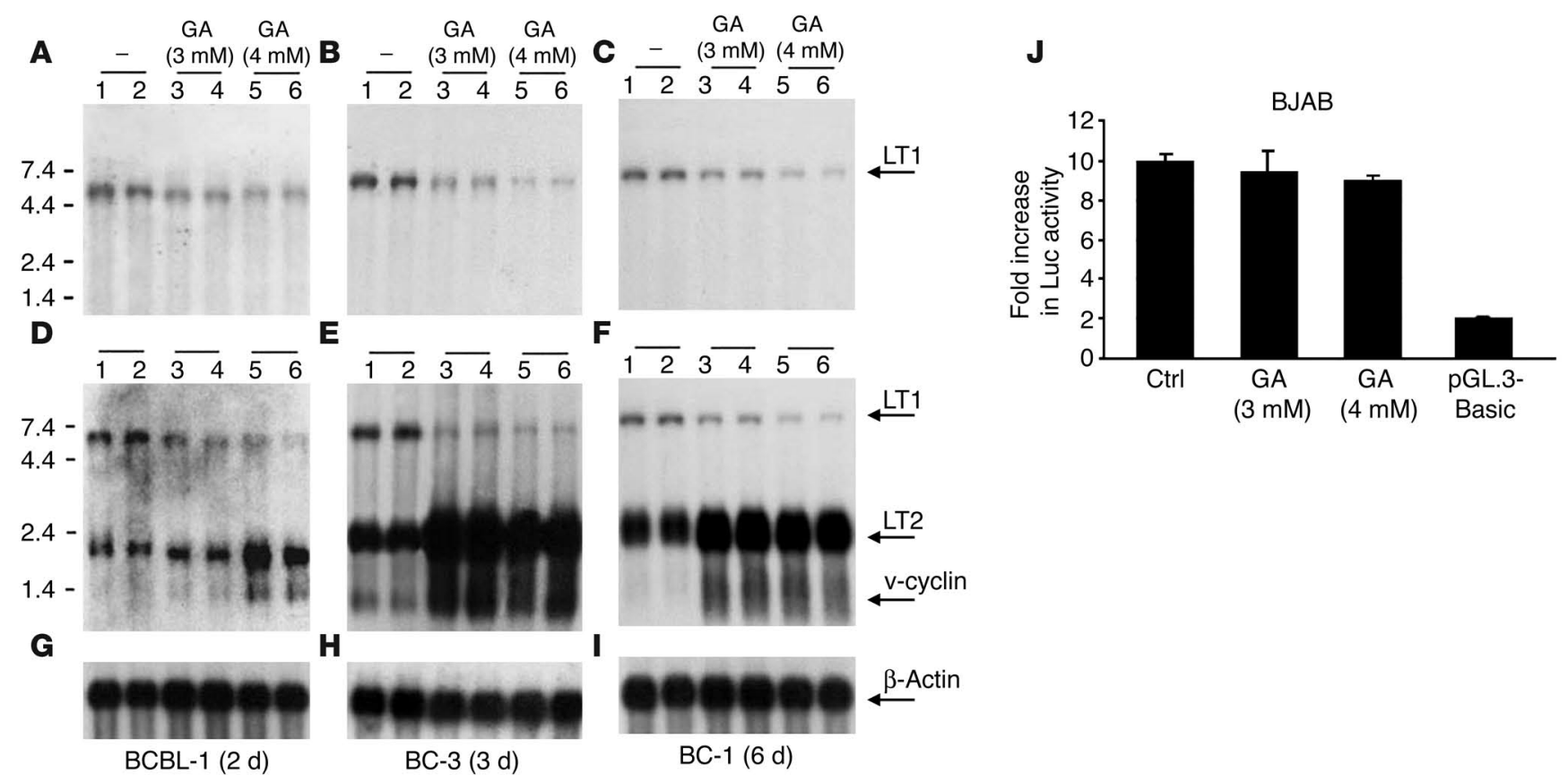

Figure 2

Latent transcript levels and luciferase assay. (A-I) Northern blot analysis. mRNA was extracted from BCBL-1 (A, D, and G), BC-3 (B, E, and H), and BC-1 (C, F, and I) cells untreated or treated with 3 or 4 mM GA. Samples were loaded in duplicate. RNA was hybridized with ORF73 probe $(\mathbf{A}-\mathbf{C})$ to detect LT1 transcripts and with ORF72 (v-cyclin) probe (D-F) to detect LT1 and LT2 transcripts. $\beta$-actin probe was used as a control (G-I). (J) Luciferase assay. First bar indicates the levels of luciferase activity in BJAB cells transfected with the LT1/LT2 promoter cloned in pGL.3-Basic (control) without GA treatment. Second and third bars indicate the levels of luciferase in BJAB cells transfected with the LT1/LT2 promoter cloned in pGL.3-Basic and then treated with 3 or $4 \mathrm{mM} \mathrm{GA}$. Fourth bar indicates the levels of luciferase in BJAB cells transfected with only the vector pGL.3-Basic (background). The $y$ axis indicates the fold increase relative to the background levels. Values are the averages of 3 experiments with 3 repeats per sample. Data represent the mean \pm SEM. Ctrl, control.

$1 \times 10^{5}$ to $2 \times 10^{5}$ cells $/ \mathrm{ml}$ for HUVEC cells. There were no significant differences in the growth curves of all KSHV-negative cells (Ramos, SLK, HUVEC, keratinocytes, and BJAB), either untreated or treated with 2, 3, or $4 \mathrm{mM} \mathrm{GA}$, and their proliferation rates were comparable to that of untreated KSHV-infected cells. On the other hand, in the presence of $4 \mathrm{mM}$ GA, the number of viable $\mathrm{KSHV}$-infected cells decreased from $4 \times 10^{5}$ to less than $1 \times 10^{4}$ cells $/ \mathrm{ml}$ after 4 days for BCBL- 1 cells and from $4 \times 10^{5}$ to $1-2 \times 10^{5}$ cells $/ \mathrm{ml}$ after 6 days for BC- 3 and BCP- 1 cells. In KS2616, a KSHV-infected HUVEC cell culture in monolayer, the number of viable cells decreased from $5 \times 10^{4}$ to $2 \times 10^{4}$ cells $/ \mathrm{ml}$ after 6 days of treatment with 2, 3, or $4 \mathrm{mM} \mathrm{GA}$. Within 8-9 days, all KSHV-infected cells died. In BC-1 and BC- 2 cells, which are KSHV- and EBV-positive, the decline of the growth curve was less dramatic; nevertheless, both cell lines died within 15 days of treatment. The coinfection with $\mathrm{EBV}$ is most likely responsible for the delay of BC-1 and BC-2 cell death; in fact, CB33 cells, which are infected with only EBV, died within 15 days. This can be explained by GA having effect on EBV itself, as already reported (33). In our experiments, the $\mathrm{ED}_{50}$ varied between 2 and $3 \mathrm{mM}$ GA, depending on the doubling time of the cell culture, with the exception of $0.5 \mathrm{mM}$ for KS2616 cells.

Data obtained by counting the cells with trypan blue were also confirmed by ${ }^{3} \mathrm{H}$-thymidine incorporation assays (data not shown). The results shown in Figure 1 indicate that GA selectively promotes cell death in latent KSHV-infected cells and that, at the active concentration, it is not toxic for uninfected cells.
$G A$ alters latent gene transcript levels. Because KSHV latent genes are responsible for viral persistence, we determined the levels of KSHV latent gene transcripts in GA-treated cells by Northern blot analysis of 4 different KSHV-infected B cells, untreated or treated with 3 or $4 \mathrm{mM} \mathrm{GA}$, the two active and nontoxic concentrations (Figure 2, A-I). We used 2 cell lines infected with KSHV (BC-3 and BCBL-1) and 2 coinfected with KSHV and EBV (BC-1 and BC-2) to show that the presence of EBV was not interfering with GA activity. Because $90 \%$ of the human population is EBV-positive, it was important to demonstrate that GA was effective on latent KSHV infection despite the presence of EBV.

On the basis of the growth curves, BCBL- 1 cells were treated with GA for 2 days, BC- 3 cells, for 3 days, and BC- 2 and BC- 1 cells, for 6 days, because each cell type required different times of treatment to observe the same effect in transcript levels. The filters were hybridized with a probe specific for ORF73 (LANA) that detects LT1 transcripts only. In all GA-treated cells, LT1 transcripts markedly decreased in a dose-dependent manner (Figure 2, A-C). To determine whether LT2 transcripts also decreased during GA treatment, 2 additional blots were hybridized with 2 different probes specific for K13 (v-FLIP) or for ORF72 (v-cyclin). The findings confirmed the decrease of LT1 transcript, but, surprisingly, an increase of LT2 transcripts was detected (Figure 2, D-F). Similar results were obtained in BC-2 cells (data not shown). Transcript levels of $\beta$-actin were similar in both untreated and GA-treated cells (Figure 2, G-I).

The low levels of LANA transcripts in the cells treated with GA could suggest a decreased activity or no activity of the LT1/LT2 

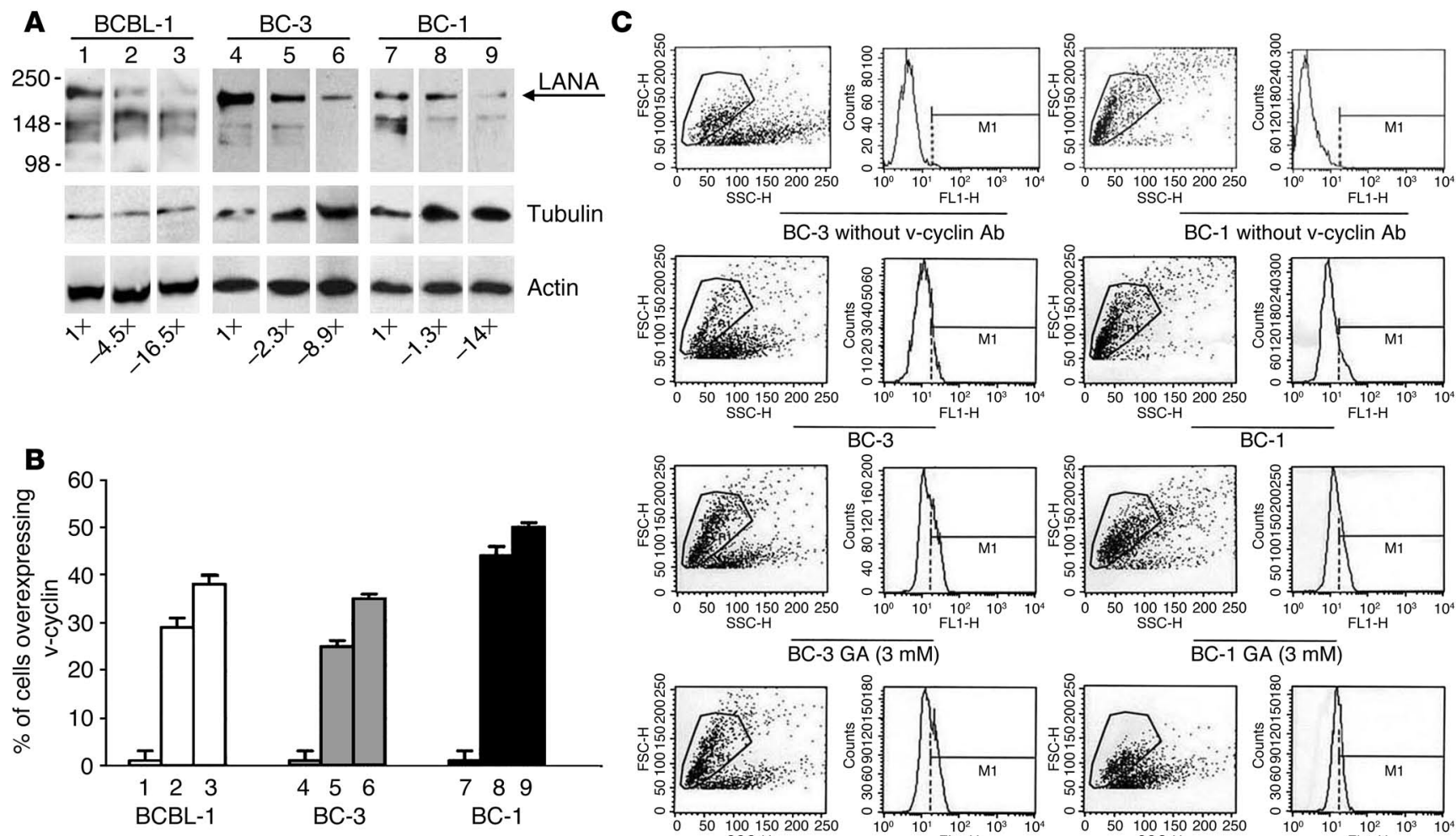

BC-1
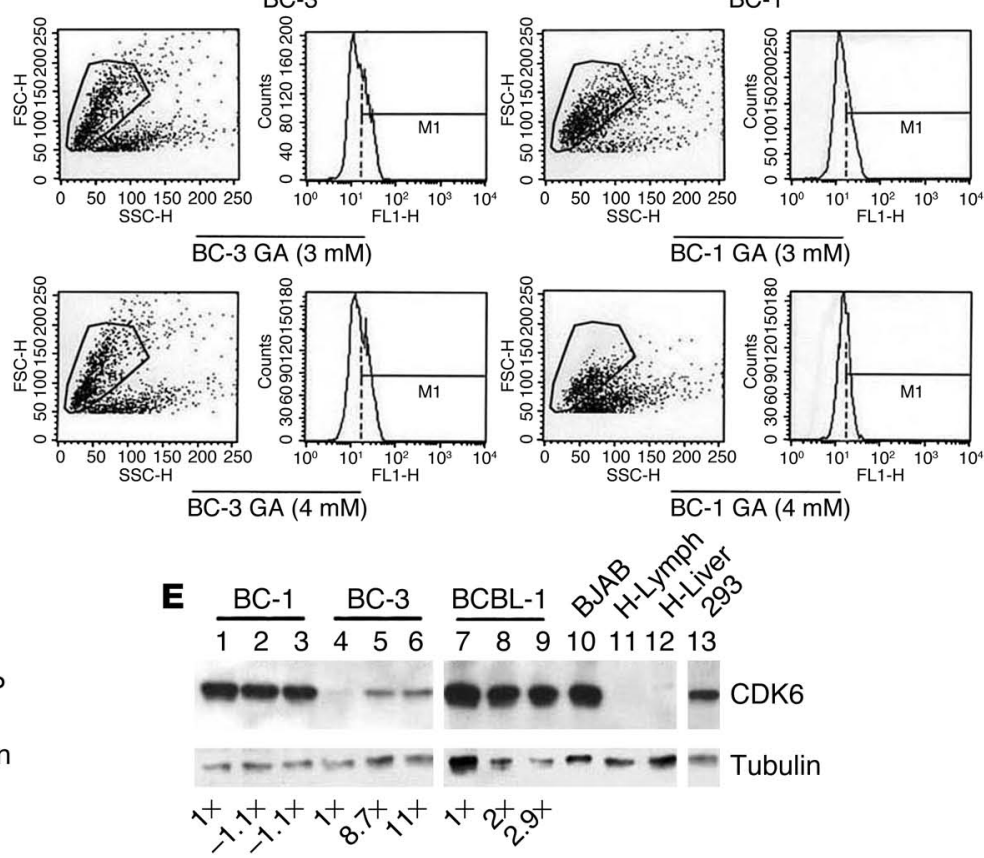

Figure 3

Protein expression. (A) LANA levels of untreated and GA-treated cells. Tubulin and actin were used as controls. Lanes 1, 4, and 7: untreated cells; lanes 2, 5, and 8: cells treated with $3 \mathrm{mM} \mathrm{GA}$; lanes 3, 6, and 9: cells treated with $4 \mathrm{mM} \mathrm{GA}$. One representative experiment is shown. The SD from 3 independent experiments is 0.22 . (B) FACS analysis of untreated and GA-treated cells stained with FITC-conjugated anti-v-cyclin. Because v-cyclin is constitutively expressed by all KSHV-infected cells, the graph shows the percentage of cells overexpressing v-cyclin. Values of GA-treated BCBL-1, $\mathrm{BC}-3$, and BC-1 cells are relative to those of the same untreated cells, arbitrarily set to 1. Columns 1, 4, and 7: untreated cells; columns 2, 5, and 8: cells treated with $3 \mathrm{mM} \mathrm{GA}$; columns 3, 6, and 9: cells treated with $4 \mathrm{mM} \mathrm{GA}$. Values are the average of 3 experiments with 3 repeats per sample. Data represent the mean \pm SEM. (C) FACS profiles of BC-3 and BC-1 cells, untreated and treated with GA and stained with FITC-conjugated anti-v-cyclin. BCBL-1 profiles were similar to those of BC-1 and BC-3 (not shown). One representative experiment is shown. (D) v-FLIP expression. Immunoblotting of cells untreated and treated with GA. Tubulin was used as control. Lane 1: BJAB cells (negative control); lanes 2, 5, and 8: untreated cells; lanes 3, 6, and 9: cells treated with $3 \mathrm{mM} \mathrm{GA}$; lanes 4, 7, and 10: cells treated with $4 \mathrm{mM} \mathrm{GA}$. One representative experiment is shown. (E) CDK6 expression. Immunoblotting of BC-1, BC-3, BCBL-1, BJAB, primary human lymphocytes (H-Lymph), primary human hepatocytes (H-Liver), and 293 cells. Lanes 1, 4, and 7: untreated cells; lanes 2, 5, and 8: cells treated with 3 mM GA; lanes 3, 6, and 9: cells treated with 4 mM GA. Tubulin was used as control. One representative experiment is shown. The SD from 3 independent experiments is \pm 0.20 .

promoter. On the other hand, the high abundance of v-cyclin and v-FLIP transcripts, which originate from the same promoter, excludes the possibility that the promoter is inactive and suggests that it has increased activity. To determine whether GA treatment affects LT1/LT2 promoter activity, we assessed its ability to drive expression of a reporter gene in transiently transfected BJAB cells. The results showed that luciferase expression levels were similar in cells untreated or treated with GA, indicating that there was no alteration in promoter activity during the treatment (Figure 2J).
Expression patterns of LANA, v-cyclin, and v-FLIP proteins. We then assessed whether the expression of LANA, v-cyclin, and v-FLIP proteins mirrored the altered transcription pattern observed in the Northern blot analysis. Their expression was measured by Western blot and FACS analysis of BCBL-1, BC-3, and BC- 1 cells untreated and treated with GA for 2, 3, and 6 days, respectively. As expected, LANA protein was detected in all untreated KSHV-infected B cells $(3,6)$. In contrast, LANA clearly decreased when the same cells were treated with GA (Figure 3A). To determine whether the effect of 
A
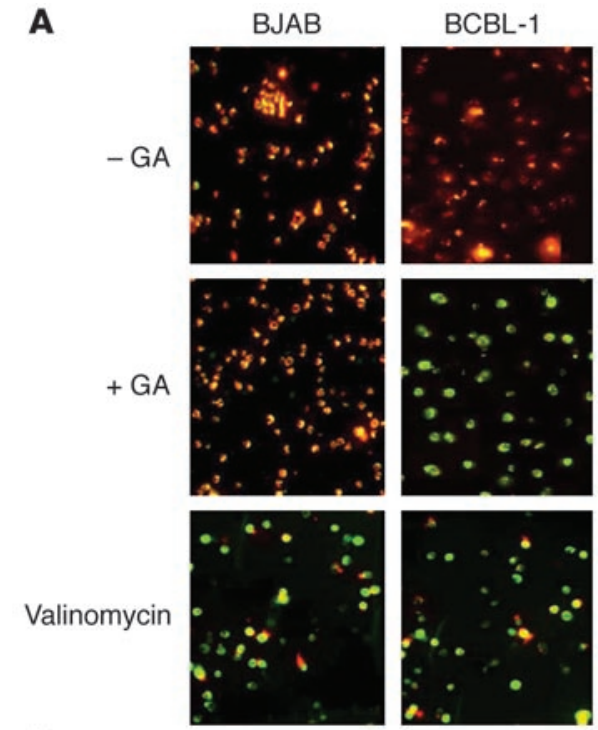

$$
\text { B }
$$
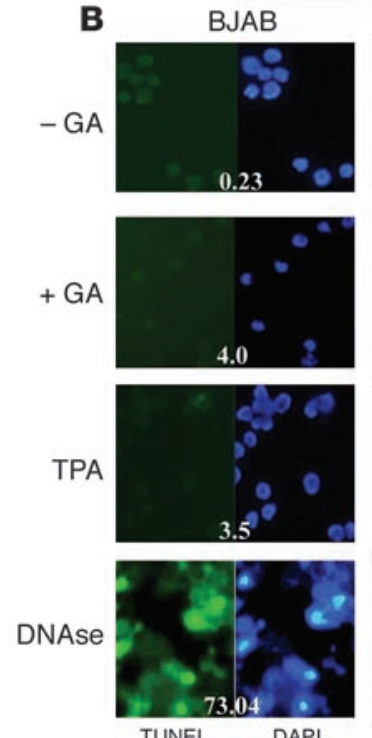

TUNEL

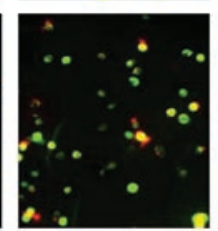

BCBL-1
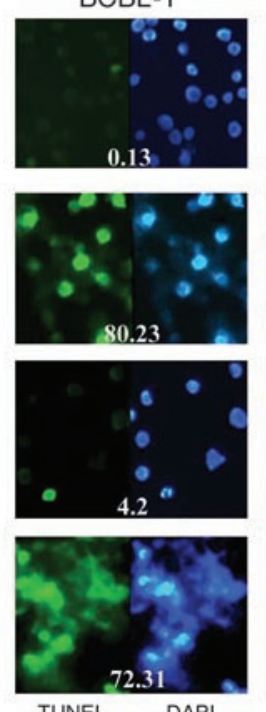

TUNEL
$\mathrm{BC}-3$
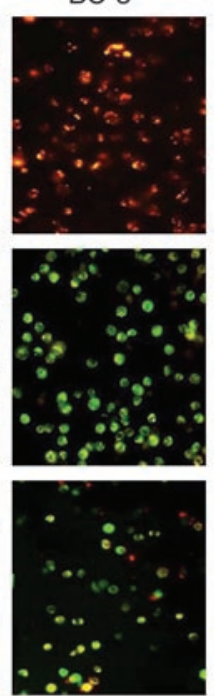

$\mathrm{BC}-3$
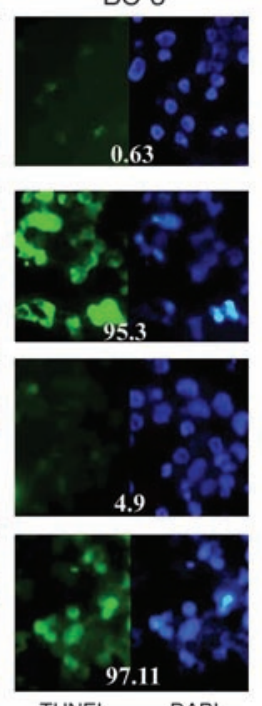

TUNEL
BC-1
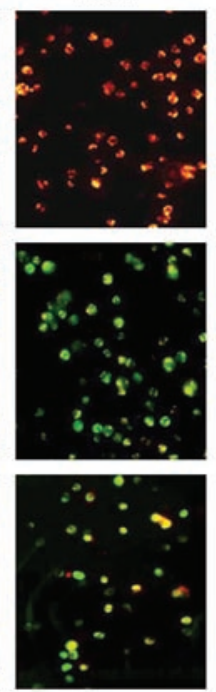

$\mathrm{BC}-1$
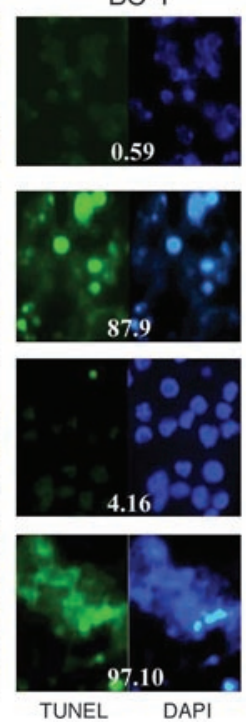

\section{Figure 4}

Apoptosis signaling. (A) Analysis of mitochondrial membrane potential disruption in BJAB, BCBL-1, $B C-3$, and BC-1 cells untreated or treated for 4 days with $4 \mathrm{mM} \mathrm{GA}$. In healthy cells, mitochondria appear red, as shown in untreated cells and in GA-treated BJAB cells. In dying cells with disrupted potential, the dye appears green, as shown in BCBL-1, BC-3, and BC-1 cells treated with GA. Valinomycin (100 nM) was used as positive control. (B) TUNEL assay to detect chromatin condensation in BJAB, BCBL-1, BC3 , and $B C-1$ cells after 4 days of treatment with 4 mM GA (green). Cells were counterstained with DAPI to localize the nuclei (blue). TPA was used as a negative control to show that lytic cycle induction does not promote chromatin condensation. Numbers indicate the percentage of TUNEL-positive cells in the same culture determined by flow cytometric analysis.
GA on LANA was reversible, BCBL-1, BC-3, and BC-1 cells were treated with $4 \mathrm{mM}$ GA. Each cell culture was treated with GA for $2,3,4,5$, and 6 days, washed, and maintained in culture with fresh medium without GA for as long as 8 days in total; then a Western blot analysis was performed. In BC-3 and BC- 1 cells, the effect on LANA was reversible up to 4 days of treatment, whereas in BCBL-1 cells, which are more sensitive to GA, the effect was reversible up to 3 days (data not shown).

To better identify differences in v-cyclin expression, we performed FACS analyses of $4 \mathrm{KSHV}$-infected B cells untreated or treated with GA. We could not measure v-cyclin expression by Western blot analysis because no commercial antibodies against v-cyclin are available for this method. Because all KSHV-infected B cells constitutively express $\mathrm{v}$-cyclin, we assessed the percentage of cells overexpressing v-cyclin during GA treatment, which was $35-50 \%$ of all GA-treated cells. Figure 3B shows the results from 3 cell lines (BCBL-1, BC-3, and $\mathrm{BC}-1$ ) after 2, 3, and 6 days of treatment. Comparable results were obtained in BC- 2 cells (data not shown). Profiles of BC-3 and BC- 1 cells are shown in Figure 3C; BCBL-1 profiles are not shown because they are similar to those of BC-3 cells. Interestingly, no difference in v-FLIP expression was observed between GA-treated or untreated KSHV-infected B cells (Figure 3D). v-FLIP is known to block Fas-mediated apoptosis $(27,28)$ in latent KSHV-infected cells. However, in our experiments the same cells undergo apoptosis when treated with GA. This indicates that v-FLIP is not preventing apoptosis but is nonetheless expressed.

Previous studies have reported that overexpression of v-cyclin in cells with elevated levels of cdk6 promotes apoptosis, and that cellular Bcl-2, as well as v-FLIP, does not inhibit v-cyclin/cdk6-induced apoptosis $(18,21)$. Therefore, we analyzed the levels of cdk6 expression in latent KSHV-infected BC-1, BC-3, and BCBL-1 cells. Normal human lymphocytes, normal liver cells, 293 human epithelial kidney cells, and BJAB cells were used as control cells (Figure 3E). Western blot analysis showed high levels of cdk6 in both GA-treated and untreated KSHV-infected B cells, except in untreated BC-3 cells. However, in the same cells, cdk6 expression increased 8- to 11-fold following treatment with GA. Therefore, BC-3 cells were induced to undergo apoptosis. Among the controls, 293 and BJAB cells showed 


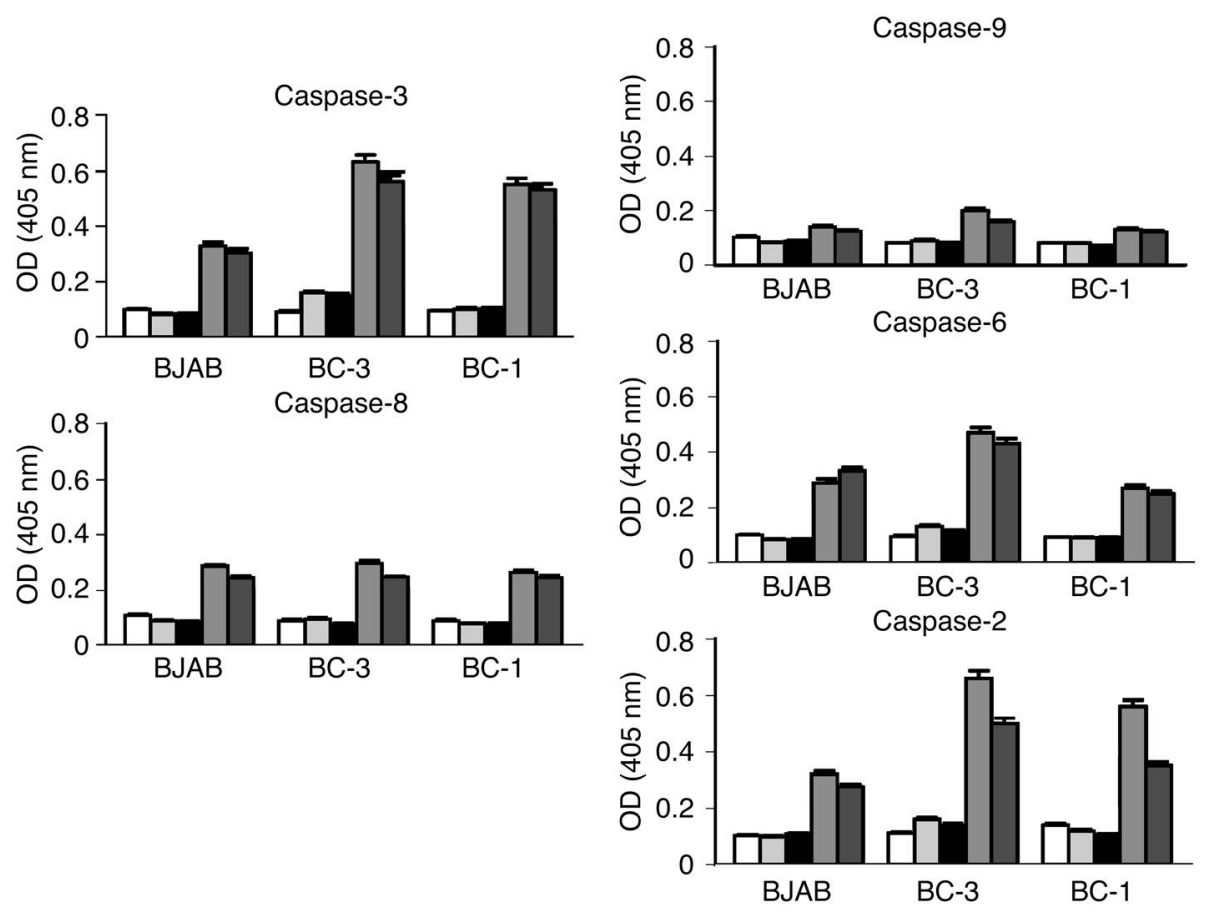

Figure 5

Detection of caspase cascade activation. BJAB, BC-3, and BC-1 cells were treated with $4 \mathrm{mM} \mathrm{GA}$ for 4 days, and $100 \mu \mathrm{g}$ of protein from each sample were analyzed with Apotarget Caspase Colorimetric Protease Assay (BioSource international) in accordance with the manufacturer's instructions. As positive control, caspase activation was induced by incubating BJAB, BC-1, and BC-3 cells with $10 \mu \mathrm{g} / \mathrm{ml}$ camptothecin (Sigma-Aldrich) for 6 hours or with $1 \mu \mathrm{M}$ staurosporine for 3 hours. Absorbance was measured at $405 \mathrm{~nm}$. Results are the averages of 3 experiments with 3 repeats per sample. White bars: untreated cells; light gray bars: cells treated with $3 \mathrm{mM} \mathrm{GA}$; black bars: cells treated with $4 \mathrm{mM} \mathrm{GA}$; medium gray bars: cells treated with staurosporine; dark gray bars: cells treated with camptothecin. high levels of cdk6. However, BJAB cells did not undergo apoptosis when treated with GA, because they are KSHV-negative and do not express v-cyclin. These results suggest that overexpressed v-cyclin/ cdk6 complex might contribute to the cell death observed in KSHVinfected B cells when treated with GA.

Latency deregulation and apoptosis signaling. Next we investigated the biological consequence of altered latent gene expression in KSHVinfected B cells treated with GA. Disruption of mitochondrial membrane potential is one of the first intracellular changes following the onset of apoptosis. As a result, proteins, such as cytochrome $c$ and AIF, that are normally localized in the mitochondrial intermembrane space translocate to the nucleus, triggering a cascade of catabolic reactions that lead to apoptosis (41). When released, cytochrome $c$ with Apaf-1 and procaspase- 9 compose the "apoptosome" (10), which activates the caspase cascade and apoptosis. Indeed, when treated with GA, KSHV-infected B cells showed a typical pattern of cells with disrupted mitochondrial membrane (Figure 4A, green color) and numerous apoptotic cells with condensed or fragmented chromatin (Figure 4B, TUNEL assay). A FACS analysis performed to determine the percentage of TUNEL-positive cells showed that $80-95 \%$ of KSHVinfected cells treated with GA were apoptotic. This percentage is markedly higher than the percentage of cells overexpressing v-cyclin during GA treatment (35-50\%, shown in Figure 3B), indicating that $\mathrm{v}$-cyclin is not solely responsible for the observed apoptosis. Neither mitochondrial membrane alteration nor DNA condensation was detected in uninfected B cells treated with GA or in cells treated with a 12-O-tetradecanoyl-phorbol-13-acetate (TPA), which promotes the switch from latent to lytic viral cycle and was used to rule out any involvement of lytic gene expression in the observed apoptosis.

Usually, permeabilization of the mitochondrial membrane promotes caspase-cascade activation that leads to DNA fragmentation. Therefore, we examined by ELISA the activation of the caspase cascade in KSHV-infected B cells either untreated or treated with GA. No activation was observed in any sample (Figure 5), suggestive of a caspase-independent pathway of apoptosis. Therefore, we focused our attention on AIF, a mitochondrial oxidoreductase that, in stress conditions, translocates from the mitochondria to the nucleus, leading to DNA loss and chromatin condensation; alterations in cell death are observed when caspases are inhibited $(11,12)$. To detect AIF translocation, we performed an immunofluorescence analysis in KSHV-infected B cells after 4 days of GA treatment. A cytoplasmic pattern, characteristic of mitochondrial AIF, was noticeable in untreated KSHV-infected B cells as well as in KSHV-negative cells (BJAB) both untreated and GA-treated, whereas a diffuse nuclear staining, signifying translocation to the nucleus, was detected in KSHV-infected B cells treated with GA (Figure 6). Therefore, we can assert that GA induced an alteration of mitochondrial membrane potential with AIF translocation to the nucleus and DNA fragmentation exclusively in KSHV-infected B cells. Taken together, these results suggest that the change in the expression pattern of KSHV latent genes (LANA downregulation and v-cyclin upregulation) is responsible for the apoptotic effects.

p53 activation and oxidative stress. Several studies reported that $\mathrm{v}$-cyclin overexpression promotes cell cycle progression and apoptosis $(18,21)$, while others showed that LANA prevents apoptosis (6) by inactivating p53. Loss of p53 function is a frequent and important occurrence in the genesis and progression of many human malignancies. In response to DNA damage, p53 is activated by phosphorylation at Ser15 or Ser20, which in turn induces cell cycle arrest in $G_{1}$ to allow DNA repair. If repair is unsuccessful, p53 initiates apoptosis. Therefore, LANA downregulation in KSHV-infected B cells might lead to p53 reactivation and consequent triggering of apoptosis signals. To verify this hypothesis, we compared the levels of nonphosphorylated and phosphorylated p53 in KSHV-infected and uninfected 
BJAB
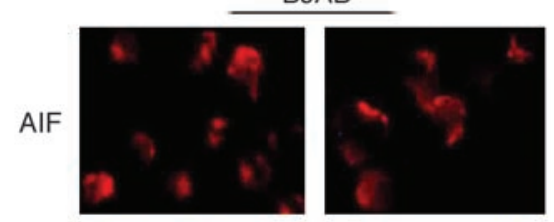

DAPI
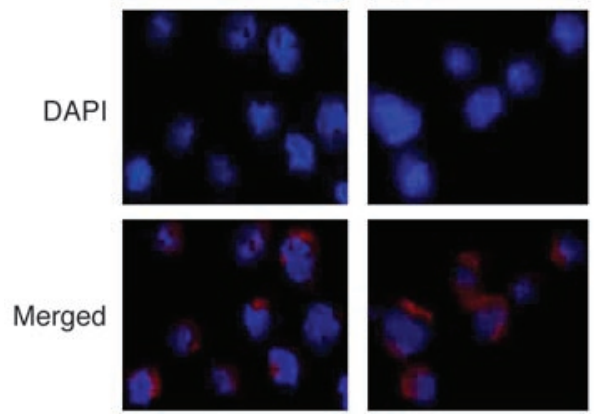

$-\mathrm{GA}$

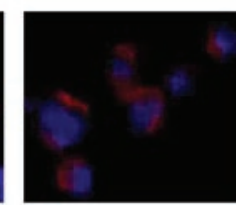

$+\mathrm{GA}$
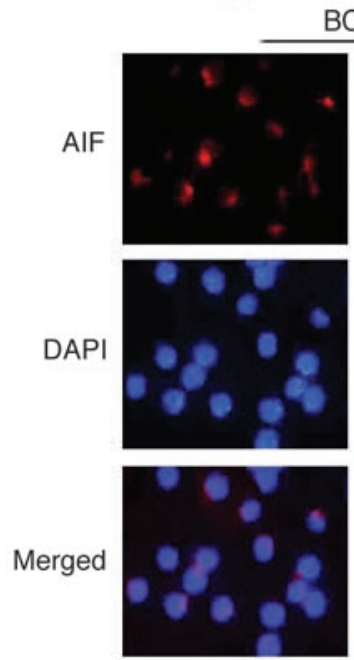

$-\mathrm{GA}$
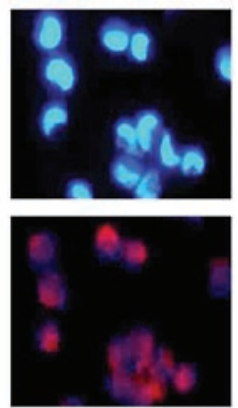

$+\mathrm{GA}$
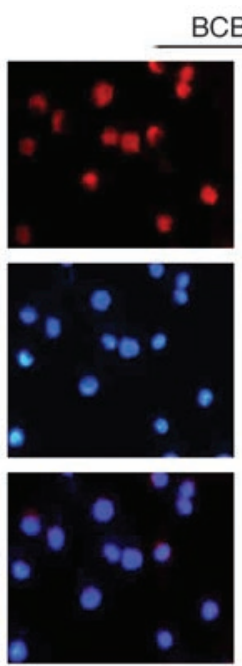

$-\mathrm{GA}$
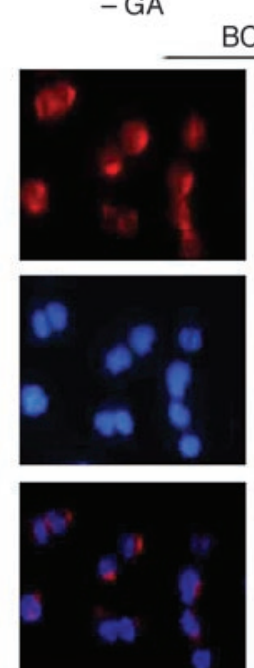

$-\mathrm{GA}$
BCBL-1
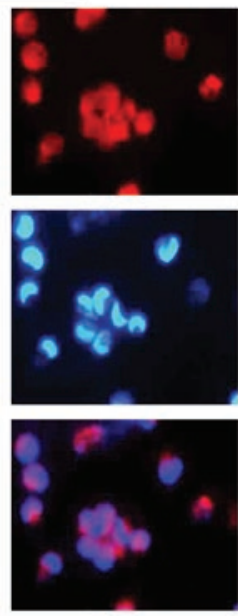

$+\mathrm{GA}$

$\mathrm{BC}-1$

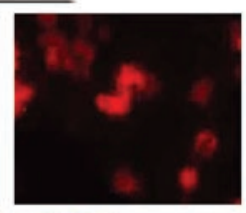

Figure 6

AIF expression. Immunofluorescence analysis was performed with BJAB, BCBL-1, BC-3, and $B C-1$ cells untreated or treated with $4 \mathrm{mM}$ GA for 4 days stained with a polyclonal antiAIF (red). Nuclei were localized with DAPI (blue).

B cells, both untreated and treated with GA. High levels of p53 phosphorylated at Ser15 were detected only in KSHV-infected B cells treated with GA. On the contrary, the levels of nonphosphorylated p53 were higher in untreated KSHV-infected cells than in those treated with GA (Figure 7A). To confirm that downregulation of LANA by GA was responsible for p 53 phosphorylation and activation, we transfected BC- 3 cells with a mammalian expression vector encoding LANA under control of the CMV promoter (pLPCX/LANA). After 24 hours, cells were treated with $4 \mathrm{mM} \mathrm{GA}$ for 4 days. A Northern blot analysis of transfected BC-3 cells was performed to confirm the presence of the 3.5-kb LANA transcript (Figure 7B). A Western blot analysis showed very low levels of phosphorylated p 53 and conversely high levels of nonphosphorylated p53 when LANA was overexpressed in BC-3 cells treated with GA (Figure 7B). These results confirm that LANA was responsible for abrogating p 53 phosphorylation, as already shown by Friborg et al. (6).
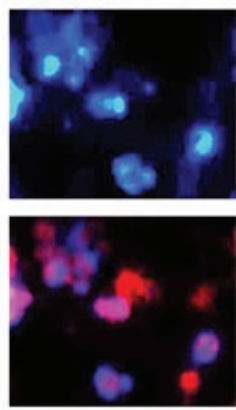

$+\mathrm{GA}$
An important step of p53-induced apoptosis is the formation of ROS (42), including $\mathrm{H}_{2} \mathrm{O}_{2}, \mathrm{O}_{2}$, and $\mathrm{OH}$. Catalase is a principal scavenger of $\mathrm{H}_{2} \mathrm{O}_{2}$ in cellular systems, and its activity increases with the increase of $\mathrm{H}_{2} \mathrm{O}_{2}$ (43). To measure ROS production, we examined catalase activity in KSHV-infected B cells, either untreated or treated with GA (Figure 7C). Catalase activity increased 4-fold (BC-3), 2-fold (BCBL-1), or 3 -fold (BC-1) in GA-treated KSHVinfected cells in comparison with untreated KSHV-infected cells and KSHV-negative cells (BJAB) either untreated or treated with GA.

Next we analyzed by FACS the cell cycle distribution of KSHV-infected and uninfected B cells both untreated and treated with GA. After 6 days, $99 \%$ of KSHVinfected $B$ cells treated with GA were blocked in $\mathrm{G}_{1}$ in comparison with $25-50 \%$ of untreated KSHV-infected cells and with uninfected cells either GA-treated or untreated (Figure 7D). These results confirmed that downregulation of LANA expression restores $\mathrm{p} 53$ function, which in turn induces cell cycle arrest of the latent KSHV-infected cells at the $\mathrm{G}_{1}$ checkpoint.

\section{Discussion}

Latency is a complex process in which host cell factors, cellular repressor molecules, and viral proteins are strictly connected, and each plays a role in this alternative infection pathway. It is a very difficult task to disrupt the close relationship between the latent herpesvirus genome, its latent proteins, and infected cells. Here we demonstrate that it is possible to interfere with this delicate balance by altering the expression pattern of the viral latent genes with a small molecule (GA). We show that GA selectively promotes cell death in latent KSHV-infected cells, and it is not toxic at active concentrations (Figure 1). During the treatment with GA we found that the levels of KSHV latent transcripts were altered: LT1 decreased and LT2 increased (Figure 2, A-F). These data suggest that GA does not affect the rate of transcription from the LT1/LT2 promoter. In fact, although the relative abundance of LT1 message decreased with treatment, this change was counterbalanced by an increase in the amount of LT2 message so that the overall level of latent transcripts remained constant. Indeed, luciferase expression levels driven by LT1/LT2 promoter were similar in untreated cells and cells treated with GA, indicating that there was no alteration in promoter activity during the treatment (Figure 2J). Nevertheless, the levels of LANA and v-cyclin/v-FLIP transcripts, which originate from this promoter, are changed during the treatment. These findings suggest that $\mathrm{GA}$ interferes with the splicing of the polycistronic messenger, which encodes LANA, v-cyclin/v-FLIP (LT1), or v-cyclin/v-FLIP (LT2) alternatively. Experiments to confirm this hypothesis are ongoing. 
A

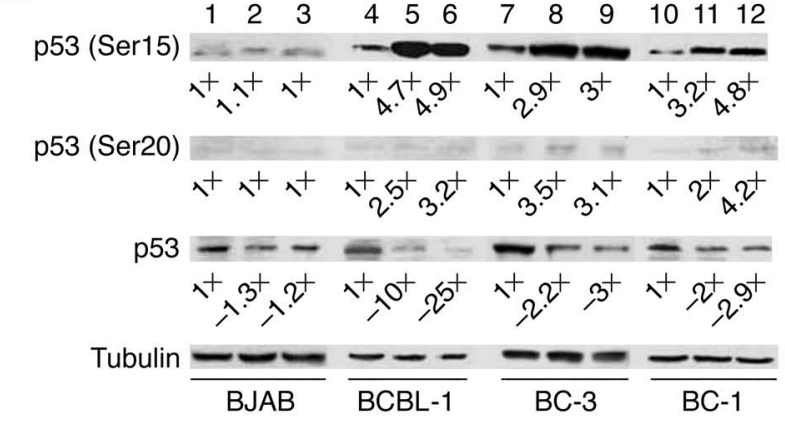

B

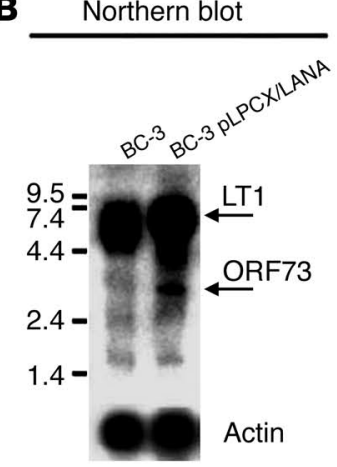

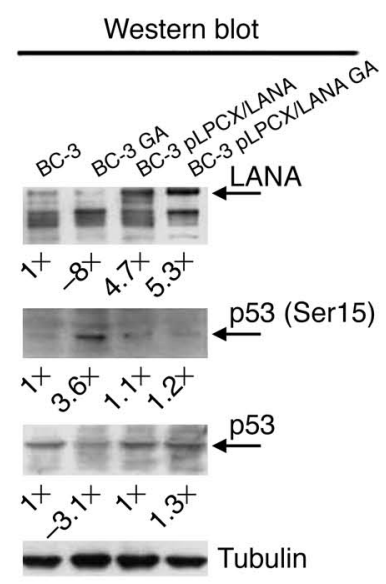
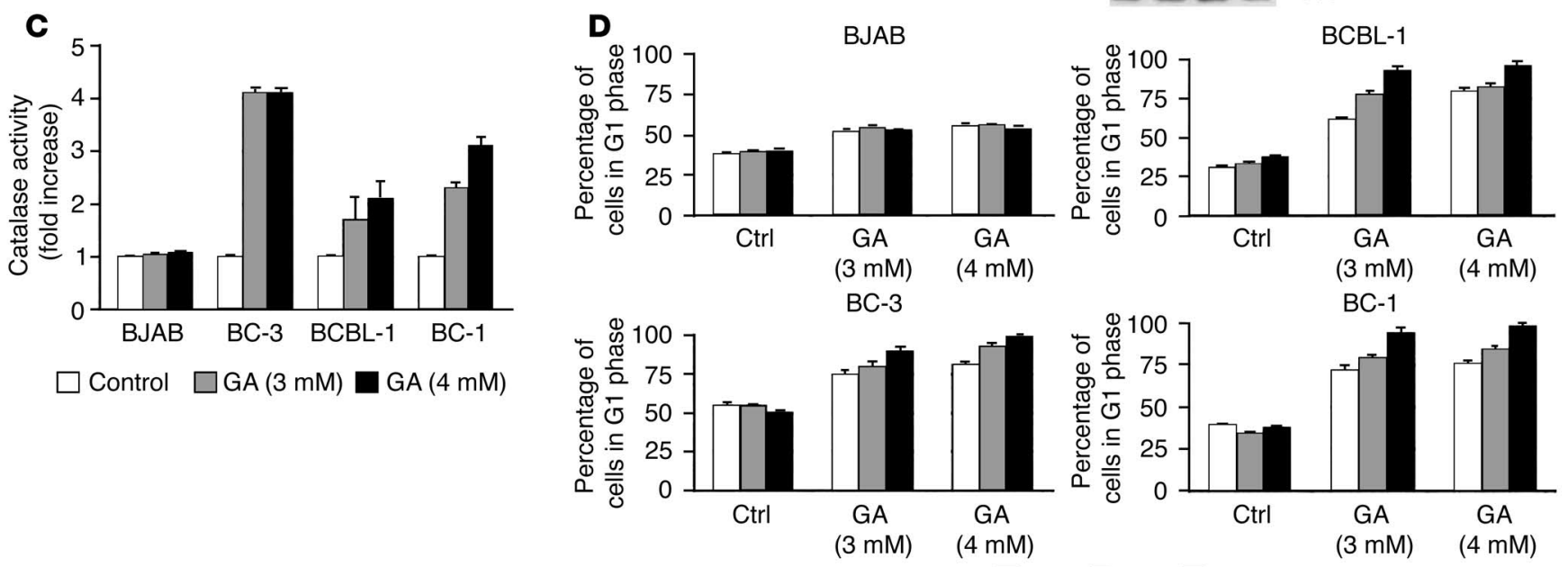

$\square 2 \mathrm{~d} \square 4 \mathrm{~d} \square 6 \mathrm{~d}$

Figure 7

Effects of p53 reactivation. (A) Levels of p53 and phosphorylated p53 in cells treated for 4 days with 3 or 4 mM GA. Cells were immune-stained with anti-p53 Ser15 or Ser20 to detect phosphorylated p53 at the specific Ser and with anti-p53 to detect nonphosphorylated p53. Tubulin was used as control. Lanes 1, 4, 7, and 10: untreated cells; lanes 2, 5, 8, and 11: cells treated with 3 mM GA; lanes 3, 6, 9, and 12: cells treated with $4 \mathrm{mM} \mathrm{GA}$. One representative experiment is shown. The SD from 3 independent experiments is 0.25 . (B) LANA overexpression in BC-3 cells transfected with pLPCX/LANA and GA-treated to abrogate p53 phosphorylation. Northern blot shows 6-kb LT1 transcript and 3.5-kb ORF73 (LANA) transcript, encoded by CMV promoter of pLPCX vector. Western blot shows the expression levels of LANA, phosphorylated p53, and p53. One representative experiment is shown. The SD from 3 independent experiments is 0.24. (C) Catalase assay in cells untreated or GA-treated for 4 days. Catalase activity was measured by absorbance at $595 \mathrm{~nm}$. Absorbance of the treated cells is relative to that of the untreated cells arbitrarily set to 1 (control). Results are the averages of 3 experiments with 3 repeats per sample. Data represent the mean \pm SD. (D) FACS analysis. Graphs show the percentage of cells blocked in G1 phase after 2, 4, or 6 days without (Ctrl) or with 3 or 4 mM GA. DNA content was quantified by staining the cells with propidium iodide. Data represent the mean \pm SD.

While LANA and v-cyclin proteins reflected the altered transcription pattern (downregulated LT1/LANA and upregulated LT2/ v-cyclin) (Figure 3, A-C), the expression levels of v-FLIP, also translated from the LT2 message, remained unchanged (Figure 3D). $\mathrm{v}$-FLIP has been shown to prevent apoptosis $(27,28)$ in latent $\mathrm{KSHV}$-infected cells. However, in the presence of GA, the same cells undergo apoptosis, indicating that v-FLIP is not preventing cell death but is nonetheless expressed at levels identical to those in untreated control cells. Taken together, these results suggest that the presence of KSHV in its latent state makes the cells susceptible to apoptosis when treated with GA and that the change in the expression patterns of LANA and v-cyclin is responsible for the apoptotic effects. As a consequence of LANA downregulation by GA, p53 transcriptional function is reactivated, as shown by its phosphorylation and cell growth arrest of latent KSHV-infected cells at the $G_{1}$ checkpoint (Figure 7). Therefore, we can assert that once p53 function is restored, its ability to induce apoptosis is also re-established, as proven by alteration of mitochondrial membrane (Figure 4A) with AIF translocation to the nucleus (Figure 6) and DNA fragmentation (Figure 4B).

These results suggest that downregulation of LANA is solely responsible for the observed cell death, and the abrogation of p53 phosphorylation in GA-treated BC-3 cells overexpressing LANA might support this hypothesis (Figure 7B). However, Ojala et al. $(18,21)$ reported that overexpressed $v$-cyclin triggers apoptosis in cells with high levels of cdk 6 by increasing caspase- 3 activity and by inactivating antiapoptotic cellular Bcl-2. We have found that KSHV-infected cells expressed high levels of cdk6 (Figure 3D) and that $35-50 \%$ of these cells overexpressed v-cyclin when treated with GA (Figure 3, B and C). On the other hand, more than $90 \%$ of 


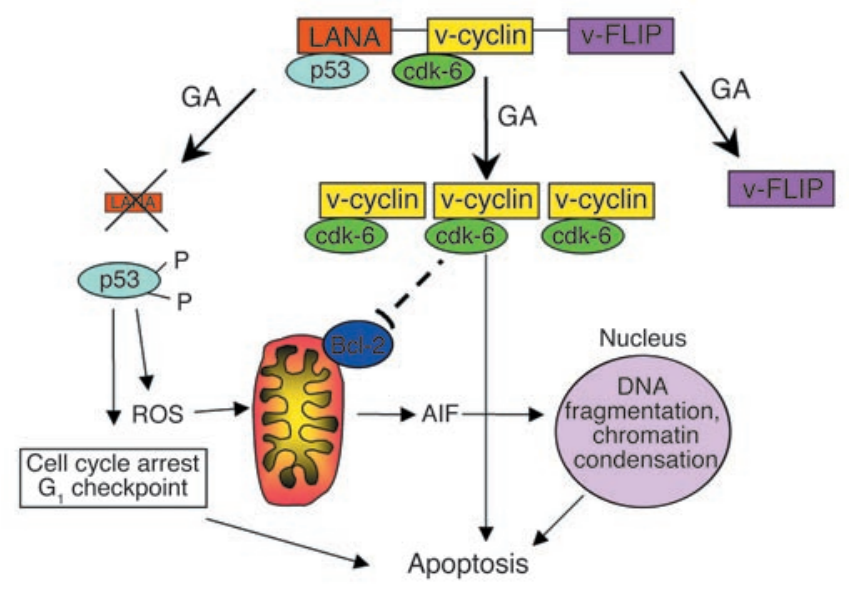

Figure 8

Schematic representation of the model of events that occur in latent KSHV-infected B lymphocytes when treated with GA. We propose that downregulation of LANA re-establishes p53 function, as shown by its phosphorylation. p53 reactivation increases the content of ROS (increased catalase activity), which leads to oxidative stress and permeabilization of the outer mitochondrial membrane. As a result, the electron flow between respiratory chain complexes is interrupted and AIF translocates to the nucleus, leading to nuclear condensation and apoptosis. Along with LANA downregulation, overexpressed v-cyclin associates with cdk6 to form a complex that inactivates antiapoptotic cellular Bcl-2 and contributes to inducing apoptosis in the KSHV-infected B cells, which express high levels of cdk6 $(18,21)$.

these same cells were undergoing apoptosis in the presence of GA, as shown by TUNEL assay (Figure 4B). Moreover, caspase- 3 and the caspase cascade were not activated (Figure 5). These data are suggestive of a limited role for v-cyclin in GA-mediated apoptosis; however, other experiments are necessary to rule out its involvement in the observed cell death. Indeed, though less crucial then LANA, overexpressed v-cyclin/cdk6 complex might contribute in inducing apoptosis in KSHV-infected cells when treated with GA by inactivating antiapoptotic cellular Bcl-2.

In Figure 8 we show a schematic representation of what we assume to occur in latent KSHV-infected B-lymphocytes when treated with GA. Briefly, GA alters latent gene expression by downregulating LANA and upregulating v-cyclin. Downregulation of LANA re-establishes p53 function, as demonstrated by its phosphorylation; reactivation of p53 increases the content of ROS, which leads to oxidative stress and permeabilization of the outer mitochondrial membrane. As a result, electron flow between respiratory chain complexes is interrupted and AIF translocates to the nucleus, leading to nuclear condensation and finally to apoptosis. In concert, overexpressed v-cyclin/cdk6 might contribute to inducing apoptosis by inactivating antiapoptotic cellular Bcl-2.

All antiherpesvirus agents described to date target only replicating virus and not latent virus. The compound we describe in this report represents what we believe to be the first example of an antiviral compound that specifically targets latent gene expression in herpesvirus-infected cells. The active concentration of GA might be considered too high to be used as an antiviral compound without modification. However, the discovery of compounds that inhibit the expression of genes required to maintaining latency opens the door for developing new strategies for antiherpesvirus agents capable of controlling, and perhaps eradicating, latent viral infections.

\section{Methods}

Cells, media, and proliferation assay. Twelve different human cell types were used. BC-3, BCBL-1, and BCP-1 cells are KSHVpositive, EBV-negative B cells (44-46). BC-1 and BC-2 cells are KSHV- and EBV-positive B cells (47). All these cells are derived from different body cavity-based lymphomas. Primary human keratinocytes were used at the second passage (Clonetics). CB33 cells are EBV-infected lymphoblastoid cells (48). Ramos cells (ATCC) are KSHV- and EBV-negative Burkitt lymphoma cells. SLK is a KSHV-negative, KS-derived cell line (49). KS2616 is a KSHV-positive cell culture, prepared in our laboratory from a KS lesion of a HIV-negative patient. This culture was used at the third passage when KSHV was still detectable (the presence of KSHV DNA was detected by PCR). HUVEC is a primary macrovascular endothelial cell culture, prepared in our laboratory from umbilical cords and used at the second passage. BJAB is a KSHV- and EBV-negative lymphoid cell line. B cells were grown in RPMI 1640 medium (Invitrogen Corp.) supplemented with $20 \%$ fetal calf serum. Cells in monolayer, HUVEC, SLK, and KS2616, were grown in M199 medium (Invitrogen Corp.) supplemented with $20 \%$ fetal calf serum, 10 ng of VEGF (Peprotech Inc.) and FGF-2 (R\&D Systems), and human keratinocytes were grown in medium for keratinocytes SFM supplemented with EGF and bovine pituitary extract (Invitrogen Corp.). Normal liver cells and 293 human epithelial kidney cells were grown in M199 medium supplemented with 10\% calf serum. GA (SigmaAldrich) was dissolved in $1 \%$ ethanol and adjusted to $\mathrm{pH} 7.2$ with $1 \mathrm{M} \mathrm{NaOH}$. Cell cultures $\left(4 \times 10^{5}\right.$ cells $\left./ \mathrm{ml}\right)$ were treated for as long as 15 days with $0.5,1,2,3,4,5$, or $6 \mathrm{mM}$ GA or with $1 \%$ ethanol. Cell viability was assessed by counting trypan blue-excluding cells on a hemocytometer every 48 hours. Cell counts were performed in triplicate.

Northern blot analysis. Total RNA was extracted from $2.5 \times 10^{7}$ cells per sample $\left(4 \times 10^{5}\right.$ cells $\left./ \mathrm{ml}\right)$ with Tri-reagent (Molecular Research Center Inc.) and mRNA was selected by Poly (A) Tract mRNA isolation kit (Promega) in accordance with the manufacturer's instructions. Northern blots were performed by loading 1 $\mu \mathrm{g}$ of mRNA onto $1.5 \%$ formaldehyde agarose gel and transferred onto a GeneScreen Plus nylon membrane. Filters were hybridized with ${ }^{32}$ P-labeled probes obtained by amplification of KSHV DNA with a set of primers already described (50). Human $\beta$-actin probe (Stratagene) was used as quantitative control.

Transfection and luciferase activity. $10^{7} \mathrm{BJAB}$ cells were transiently transfected by electroporation with $10 \mu \mathrm{g}$ of a construct, generated by cloning in front of a luciferase reporter gene (pGL.3-Basic vector; Promega) a PCR product containing sequences from $-1,450 \mathrm{bp}$ upstream of the major start site of LT1/2 promoter to $281 \mathrm{bp}$ downstream (1), at $274 \mathrm{~V}$ and $950 \mu \mathrm{F}$. Twenty-four hours after transfection, cells were treated with $3 \mathrm{mM}$ or $4 \mathrm{mM}$ GA and maintained in culture for 48 hours before assaying the reporter activity. For luciferase reporter assays, $200 \mu \mathrm{l}$ of LCCLR buffer (Promega) was added to each sample; $50 \mu \mathrm{l}$ of cell extract and $300 \mu \mathrm{l}$ of reaction buffer (25 mM glycine-glycine [pH 7.8], $15 \mathrm{mM} \mathrm{MgSO}_{4}, 1 \mathrm{mM}$ ATP [pH 7.0], $0.1 \mathrm{mg} / \mathrm{ml} \mathrm{BSA}$, and $1 \mathrm{mM} \mathrm{DTT}$ ) were mixed, added to 1 mM D-luciferin substrate (Analytical Luminescence Laboratory) and immediately assayed with a LB9507 luminometer (EG \& G Berthold). Values of the GA-treated and untreated samples are relative to the background levels (BJAB cells transfected with only pGL.3-Basic) and are the averages of 3 experiments with 3 repeats per sample. Data represent the mean \pm SEM. 
Western blots. Cells were lysed with $1 \times$ RIPA buffer. Proteins were separated by electrophoresis in $8-16 \%$ Tris-glycine gels and blotted in accordance with standard protocols. LANA was detected using a rat antibody (ABI). Antibody against v-FLIP was kindly provided by Mary Collins (University College London, London, United Kingdom). Detection of phosphorylated p53 (Ser15) or (Ser20) was performed with polyclonal antibodies, which detect only phosphorylated p53 at the specific serine (Cell Signaling Technology). Nonphosphorylated $\mathrm{p} 53$ was detected with a mAb specific for nonphosphorylated p53 (Oncogene Research Products). cdk6, $\alpha$-tubulin (polyclonal $\mathrm{Abs}$ ) and $\beta$-actin ( $\mathrm{mAb}$ ) were purchased from Santa Cruz Biotechnology Inc. Signals were visualized by enhanced chemiluminescence (ECL), and specific hybridization was quantified by ImageQuant version 1.11 software. Signal intensity was standardized to either actin or tubulin, and the effect of GA was expressed as fold decrease or increase compared with the intensity of untreated cells, rated 1. Experiments were performed 3 times and results are the average of each experiment with standard deviations.

Detection of mitochondrial membrane potential disruption. BC-1, BC-3, BCBL-1, and BJAB cells were treated with $4 \mathrm{mM}$ GA for 4 days and then analyzed with a commercial kit (DePsipher; R\&D Systems) in accordance with the manufacturer's instructions.

Caspase assay. BJAB, BC-3, and $\mathrm{BC}-1$ cells were treated with 4 $\mathrm{mM}$ GA for 4 days, and $100 \mu \mathrm{g}$ of protein from each sample were analyzed with Apotarget Caspase Colorimetric Protease Assay (BioSource International) in accordance with the manufacturer's instructions. As a positive control, caspase activation was induced by incubating BJAB, BC-1, and BC-3 cells with $10 \mu \mathrm{g} / \mathrm{ml}$ camptothecin (Sigma-Aldrich) for 6 hours or with $1 \mu \mathrm{M}$ staurosporine for 3 hours. Absorbance was measured at $405 \mathrm{~nm}$. Results are the averages of 3 experiments with 3 repeats per sample.

Catalase assay. $10^{4}$ cells per sample were washed with PBS, resuspended in $25 \mu \mathrm{l}$ of $10 \mathrm{mM}$ Tris- $\mathrm{HCl}(\mathrm{pH} 7.6)$ and $10 \mathrm{mM} \mathrm{NaCl}$, frozen and thawed twice, and spun for 3 minutes at 13,000 rpm. Catalase activity was determined using the Amplex Red Catalase Assay Kit (Molecular Probes) in accordance with the manufacturer's instructions and measured spectrophotometrically by Bradford assay (absorbance $595 \mathrm{~nm}$ ). As a control for the catalase activity, a standard curve was generated by adding $20 \mu \mathrm{M} \mathrm{H}_{2} \mathrm{O}_{2}$ to catalase concentrations of $0-5.0 \mathrm{U} / \mathrm{ml}$, in $1 \times$ Reaction Buffer for 30 minutes. Sample values are expressed as fold increase relative to absorbance of untreated cells arbitrarily set to 1 (control).

TUNEL. BC-1, BC-3, BCBL-1, and BJAB cells were treated with 4 mM GA for 4 days. Apoptotic cells were detected using the In Situ Cell Death Detection kit (Roche) in accordance with the manufacturer's instructions. DNAse I $(10 \mu \mathrm{g} / \mathrm{ml}$ for 10 minutes at room temperature) was used as positive control. TPA was used as a negative control. DAPI (blue) was used to localize the nuclei. After staining, cells were analyzed under fluorescence microscope or by FACS analysis.

FACS analysis. Cell cycle distribution was as follows: BJAB, BCBL-1, BC-3, and BC-1 cells were treated for 6 days with 3 or $4 \mathrm{mM}$ GA. Every 2 days, $3 \times 10^{6}$ cells were collected, washed with PBS, stained with $50 \mu \mathrm{g} / \mathrm{ml}$ of propidium iodide (SigmaAldrich), and then analyzed by flow cytometry.

$K S H V$-cyclin detection. Cells were fixed and permeabilized by FIX \& PERM Cell Permeabilization kit (CALTAG Laboratories Inc.) and stained with a mAb against v-cyclin (ViroStat Inc.), which was FITC-conjugated by EZ-Label FITC Protein Labeling Kit (PIERCE Biotechnology). Because v-cyclin is constitutively expressed by all KSHV-infected cells, the values indicate the percentage of cells overexpressing v-cyclin. Values of GA-treated $\mathrm{BCBL}-1, \mathrm{BC}-3$, and $\mathrm{BC}-1$ cells are relative to those of the same untreated cells arbitrarily set to 1 . As negative control, we used cells with no staining and cells stained with FITC-conjugated anti-mouse antibody (ICN/CAPPEL). Cells were gated for cell size on scatter to exclude dead cells and debris. M1 was calculated at the base (right side) of the peak of the untreated cells without staining (Figure 3C, first row).

Plasmid PLPCX/LANA construct and transfection. A product of 3,553 bp, encompassing KSHV ORF73, was generated by long RT-PCR, using RNA extracted from BC-1 cells as template. Primer LANA $3^{\prime}$ (position 123,780-123,804) immediately downstream of LANA stop codon and primer $5^{\prime}$ (position 127,303-127,831), overlapping LT1 donor and acceptor splice sites, were used to perform PCR. The resulting PCR product contains only ORF73 (LANA) without any regulatory element at either the $3^{\prime}$ or $5^{\prime}$ ends. The PCR product was cloned in the multiple cloning site of CMV-driven expression vector pLPCX (CLONTECH Laboratories, Inc.) immediately downstream of the human CMV immediate early promoter.

BC- 3 cells $\left(10^{7}\right.$ total $)$ were transiently transfected with $4 \mu \mathrm{g}$ of PLPCX/LANA using Effectene (Qiagen) in accordance with the manufacturer's instructions. After 24 hours, cells were treated with $4 \mathrm{mM}$ GA for 4 days. mRNA was extracted to perform Northern blot analysis and filter was hybridized with ORF73 probe (LANA). Proteins were extracted to perform Western blot analysis with antibodies against LANA, p53, phosphorylated p53 (Ser 15), and tubulin.

\section{Acknowledgments}

We thank Mary Collins for providing the v-FLIP antibody, Claudio Basilico and Jan Vilcek for fruitful discussions and critical reading of the manuscript, and Iqbal Ahmed and Moshe Sweet for technical help. This work was supported by the Elsa U. Pardee Foundation, the Howard Gilman Foundation, the New York University Center for AIDS Research (CFAR), and National Institute of Allergy and Infectious Diseases (NIAID) AIDS institutional training grant T32-AI07382.

Received for publication September 13, 2004, and accepted in revised form December 21, 2004.

Address correspondence to: Ornella Flore, New York University School of Medicine, 550 First Avenue, New York, New York 10016, USA. Phone: (212) 263-5313; Fax: (212) 263-7933; E-mail: ornella. flore@med.nyu.edu.

\footnotetext{
1. Sarid, R., Wiezorek, J.S., Moore, P.S., and Chang, Y. 1999. Characterization and cell cycle regulation of the major Kaposi's sarcoma-associated herpesvirus (human herpesvirus 8) latent genes and their promoter. J. Virol. 73:1438-1446.

2. Kellam, P., et al. 1999. Characterization of monoclonal antibodies raised against the latent nuclear antigen of human herpesvirus 8 . J. Virol. 73:5149-5155.
}

3. Rainbow, L., et al. 1997. The 222- to 234-kilodalton latent nuclear protein (LNA) of Kaposi's sarcoma-associated herpesvirus (human herpesvirus 8 ) is encoded by orf73 and is a component of the latency-associated nuclear antigen. J. Virol. 71:5915-5921.

4. Ballestas, M.E., Chatis, P.A., and Kaye, K.M. 1999. Efficient persistence of extrachromosomal KSHV DNA mediated by latency- associated nuclear antigen. Science. 284:641-644.

5. Cotter, M.A., 2nd, and Robertson, E.S. 1999. The latency-associated nuclear antigen tethers the Kaposi's sarcoma-associated herpesvirus genome to host chromosomes in body cavity-based lymphoma cells. Virology. 264:254-264.

6. Friborg, J., Jr., Kong, W., Hottiger, M.O., and Nabel, G.J. 1999. p53 inhibition by the LANA protein of KSHV protects against cell death. 
Nature. 402:889-894.

7. Katano, H., Sato, Y., and Sata, T. 2001. Expression of 553 and human herpesvirus-8 (HHV-8)-encoded latency-associated nuclear antigen with inhibition of apoptosis in HHV-8-associated malignancies. Cancer. 92:3076-3084.

8. Finkel, E. 2001. The mitochondrion: is it central to apoptosis? Science. 292:624-626.

9. Thornberry, N.A., and Lazebnik, Y. 1998. Caspases: enemies within. Science. 281:1312-1316.

10. Jiang, X., and Wang, X. 2000. Cytochrome c promotes caspase- 9 activation by inducing nucleotide binding to Apaf-1. J. Biol. Chem. 275:31199-31203.

11. Joza, N., et al. 2001. Essential role of the mitochondrial apoptosis-inducing factor in programmed cell death. Nature. 410:549-554.

12. Susin, S.A., et al. 1999. Molecular characterization of mitochondrial apoptosis-inducing factor. Nature. 397:441-446.

13. Bates, S., et al. 1994. Absence of cyclin D/cdk complexes in cells lacking functional retinoblastoma protein. Oncogene. 9:1633-1640.

14. Sherr, C.J. 1995. D-type cyclins. Trends Biochem. Sci. 20:187-190.

15. Swanton, C., et al. 1997. Herpes viral cyclin/Cdk6 complexes evade inhibition by CDK inhibitor proteins. Nature. 390:184-187.

16. Ellis, M., et al. 1999. Degradation of p27(Kip) cdk inhibitor triggered by Kaposi's sarcoma virus cyclin-cdk6 complex. EMBO J. 18:644-653.

17. Mann, D.J., Child, E.S., Swanton, C., Laman, H., and Jones, N. 1999. Modulation of p27(Kip1) levels by the cyclin encoded by Kaposi's sarcoma-associated herpesvirus. EMBO J. 18:654-663.

18. Ojala, P.M., et al. 2000. The apoptotic v-cyclinCDK6 complex phosphorylates and inactivates Bcl- 2. Nat. Cell Biol. 2:819-825.

19. Harris, A.W., et al. 1995. Cyclin D1 as the putative bcl-1 oncogene. Curr. Top. Microbiol. Immunol. 194:347-353

20. Resnitzky, D., Gossen, M., Bujard, H., and Reed, S.I. 1994. Acceleration of the G1/S phase transition by expression of cyclins D1 and E with an inducible system. Mol. Cell. Biol. 14:1669-1679.

21. Ojala, P.M., et al. 1999. Kaposi's sarcoma-associated herpesvirus-encoded v-cyclin triggers apoptosis in cells with high levels of cyclin-dependent kinase 6 . Cancer Res. 59:4984-4989.

22. Bieleski, L., and Talbot, S.J. 2001. Kaposi's sarcomaassociated herpesvirus vCyclin open reading frame contains an internal ribosome entry site. J. Virol. 75:1864-1869.

23. Grundhoff, A., and Ganem, D. 2001. Mecha- nisms governing expression of the v-FLIP gene of Kaposi's sarcoma-associated herpesvirus. J. Virol. 75:1857-1863

24. Low, W., et al. 2001. Internal ribosome entry site regulates translation of Kaposi's sarcoma-associated herpesvirus FLICE inhibitory protein. J. Virol. 75:2938-2945.

25. Bertin, J., et al. 1997. Death effector domain-containing herpesvirus and poxvirus proteins inhibit both Fas- and TNFR1-induced apoptosis. Proc. Natl. Acad. Sci. U. S. A. 94:1172-1176.

26. Thome, M., et al. 1997. Viral FLICE-inhibitory proteins (FLIPs) prevent apoptosis induced by death receptors. Nature. 386:517-521.

27. Djerbi, M., et al. 1999. The inhibitor of death receptor signaling, FLICE-inhibitory protein defines a new class of tumor progression factors. J. Exp. Med. 190:1025-1032.

28. Belanger, C., et al. 2001. Human herpesvirus 8 viral FLICE-inhibitory protein inhibits Fas- mediated apoptosis through binding and prevention of procaspase-8 maturation. J. Hum. Virol. 4:62-73.

29. Ito, M., et al. 1988. Mechanism of inhibitory effect of glycyrrhizin on replication of human immunodeficiency virus (HIV). Antiviral Res. 10:289-298.

30. Pompei, R., Flore, O., Marccialis, M.A., Pani, A., and Loddo, B. 1979. Glycyrrhizic acid inhibits virus growth and inactivates virus particles. Nature. 281:689-690.

31. Dargan, D.J., and Subak-Sharpe, J.H. 1992. The antiviral activity of triterpenoid compounds. Semin. Virol. 3:31-40.

32. Galt, C., Dargan, D.J., and Subak-Sharpe, J.H. 1990. In vitro studies of the antiviral range of cicloxolone sodium and identification of cell lines tolerant to the drug. Antiviral Chem. Chemother. 1:115-123.

33. Lin, J.C. 2003. Mechanism of action of glycyrrhizic acid in inhibition of Epstein-Barr virus replication in vitro. Antiviral Res. 59:41-47.

34. Cinatl, J., et al. 2003. Glycyrrhizin, an active component of liquorice roots, and replication of SARSassociated coronavirus. Lancet. 361:2045-2046.

35. Arase, Y., et al. 1997. The long term efficacy of glycyrrhizin in chronic hepatitis $\mathrm{C}$ patients. Cancer. 79:1494-1500

36. van Rossum, T.G., et al. 1999. Intravenous glycyrrhizin for the treatment of chronic hepatitis C: a double-blind, randomized, placebo-controlled phase I/II trial. J. Gastroenterol. Hepatol. 14:1093-1099.

37. Sato, H., et al. 1996. Therapeutic basis of glycyrrhizin on chronic hepatitis B. Antiviral Res. 30:171-177.
38. Tangri, K.K., Seth, P.K., Parmar, S.S., and Bhargava, K.P. 1965. Biochemical study of anti-inflammatory and anti-arthritic properties of glycyrrhetic acid. Biochem. Pharmacol. 14:1277-1281.

39. Kroes, B.H., et al. 1997. Inhibition of human complement by beta-glycyrrhetinic acid. Immunology. 90:115-120.

40. Ishiwata, S., et al. 1999. Fas-mediated apoptosis is enhanced by glycyrrhizin without alteration of caspase-3-like activity. Biol. Pharm. Bull. 22:1163-1166

41. Daugas, E., et al. 2000. Mitochondrio-nuclear translocation of AIF in apoptosis and necrosis. FASEB J. 14:729-739.

42. Polyak, K., Xia, Y., Zweier, J.L., Kinzler, K.W., and Vogelstein, B. 1997. A model for p53-induced apoptosis. Nature. 389:300-305.

43. Deisseroth, A., and Dounce, A.L. 1970. Catalase: Physical and chemical properties, mechanism of catalysis, and physiological role. Physiol. Rev. 50:319-375.

44. Arvanitakis, L., et al. 1996. Establishment and characterization of a primary effusion (body cavity-based) lymphoma cell line (BC-3) harboring Kaposi's sarcoma-associated herpesvirus (KSHV/ HHV-8) in the absence of Epstein-Barr virus. Blood. 88:2648-2654.

45. Boshoff, C., et al. 1998. Establishing a KSHV+ cell line (BCP-1) from peripheral blood and characterizing its growth in Nod/SCID mice. Blood. 91:1671-1679.

46. Renne, R., Lagunoff, M., Zhong, W.D., and Ganem, D. 1996. The size and conformation of Kaposi's sarcoma-associated herpesvirus (human herpesvirus 8) DNA in infected cells and virions. J. Virol. 70:8151-8154.

47. Cesarman, E., et al. 1995. In vitro establishment and characterization of two acquired immunodeficiency syndrome-related lymphoma cell lines (BC-1 and BC-2) containing Kaposi's sarcoma-associated herpesvirus-like (KSHV) DNA sequences. Blood. 86:2708-2714.

48. Lombardi, L., Newcomb, E.W., and Dalla-Favera, R. 1987. Pathogenesis of Burkitt lymphoma: expression of an activated $\mathrm{c}$-myc oncogene causes the tumorigenic conversion of EBV-infected human B lymphoblasts. Cell. 49:161-170.

49. Herndier, B.G., et al. 1994. Characterization of a human Kaposi's sarcoma cell line that induces angiogenic tumors in animals. AIDS. 8:575-581.

50. Flore, O., and Gao, S.J. 1997. Effect of DNA synthesis inhibitors on Kaposi's sarcoma-associated herpesvirus cyclin and major capsid protein gene expression. AIDS Res. Hum. Retroviruses. 13:1229-1233. 